\title{
TANTI PICCOLI FÜHRER? IL "PESO" DELLA DECRETAZIONE PERSONALE NEL GOVERNATORATO GENERALE DI POLONIA
}

\author{
MANY "LITTLE FÜHRERS"? THE "WEIGHT" OF PERSONAL DECREE \\ IN THE GENERAL GOVERNMENT OF POLAND
}

\author{
Emiliano Vitti
}

\author{
Dottore di Ricerca Università degli Studi di Pavia
}

\begin{abstract}
English: The system based on National Socialist political doctrine was born neither accompanied by a legal cultural basis of its own, nor with the premise of "relying on" the State in order to reorganize German social and economic life. In fact, it was the numerous National Socialist jurists who elaborated a sort of "structure of legal sources" which constituted a reserve of legal instruments to be used to reach a point of "fusion" between institutional structure and political doctrine.
\end{abstract}

The distorting effects that the new "National Socialist Law" had in the management of territories, especially in those occupied during the war, manifested themselves with greater intensity on Polish territory or, more precisely, on that portion of territory renamed Generalgouvernement für die besetzten polnischen Gebiete, more simply the General Government of Poland; both in the high levels of the Zivilverwaltung and in the administration of the local districts (Kreise), the personalisms of the officials manifested themselves strongly, also thanks to the use of forms of personal decree with different effectiveness. The objective of the "codification" of the regime thus became the object of exploitation, both by the upper echelons of the party (including Hitler), and by those who worked in the administration of the GG, especially some local officials. They, in different forms and with different intensity, fulfilled their functions as petty dictators, using juridically different and territorially limited versions of the personal decree.

Frank found himself in the position of having to justify to his Führer the leadership of the "Gau of the Vandals» and his being at the same time a man of law. Hitler expressed his contempt for the category of jurists whom he considered "traitors to the people", calling them «mentally handicapped from birth or destined to become so with the passage of time» and declared in a speech to the Reichstag on April 26, 1942 that he would have no "peace until every German was convinced that being a jurist was a shameful disgrace». Curiously, the actual exercise of power by the dictator, based on his undisputed personal and political authority, was justified on the juridical level by the doctrinal interpretation of some singular normative instruments, the Führererlasse, the maximum expression of "compromise", between justification of existing power and an attempt to codify its strength and contents in a peculiar Nazi "constitutional" structure.

They were never formally part of the German legal order, but were de facto an element approved and amply justified by the National Socialist juridical doctrine, which influenced the life of the Reich and the territories it controlled, feeding and amplifying that system

* Italian Review of Legal History, 7 (2021), n. 12, pagg. 419-449

* https://riviste.unimi.it/index.php/irlh/index

* ISSN 2464-8914 - DOI 10.54103/2464-8914/16894. Articolo pubblicato sotto Licenza CC-BY. 
of double institutional levels. which had its most chaotic realization in the GG.

The attitude of absolute sovereign on the part of the Kreishauptmänner was typical in the local districts; even from these childish behaviors and from the ostentation of a pomp which was often the result of robbery and looting, it was possible to recognize both the amateurism of a large slice of the administrative staff, and the almost unlimited possibilities caused by the use of personal instruments in the hands of district chiefs.

The command practice was based on recommended and unpunished violence: the existence of some very rare "oases of normal administration" depended exclusively on the will of the individual local leaders and on the resonance that their actions could have at the top of the state nomenclature. The way the population was treated was aimed at leaving a mark on German occupation policy and showing the efficiency of the administrators, through construction work (Aufbauarbeit) of a testimony of the historische Mission in Ostgebiet.

The objective of this paper is to relate the instrument of personal decree with the powers of which they were at the same time cause and consequence, and the behavior of the individual officials who operated in the various institutional levels taken into account, mentioning some cases of an atypical administrative entity like GG.

Keywords: Führererlasse; Zivilverwaltung; Kreise; Hans Frank; Kreishauptmann; Stadthauptmann.

Abstract Italiano: II sistema basato sulla dottrina politica nazionalsocialista non nacque accompagnato né da una base culturale giuridica propria, né con il presupposto di "appoggiarsi" allo Stato per poter riorganizzare la vita sociale ed economica tedesca. In effetti, furono i numerosi giuristi nazionalsocialisti ad elaborare una sorta di "struttura delle fonti giuridiche" che costituisse una riserva di strumenti giuridici da utilizzare per raggiungere un punto di "fusione" tra impianto istituzionale e dottrina politica di indirizzo.

Gli effetti distorsivi che il nuovo "Diritto Nazionalsocialista" ebbe nella gestione dei territori, specie in quelli occupati durante la guerra, si manifestarono con maggiore intensità sul territorio polacco, o meglio su quella porzione di territorio rinominato Generalgouvernement für die besetzten polnischen Gebiete; sia negli alti livelli della Zivilverwaltung, sia nell'amministrazione dei distretti locali (Kreise), i personalismi dei funzionari si manifestarono con forza, anche grazie all'uso di forme di decretazione personale dotate di differente efficacia.

L'obiettivo di questo scritto è porre in relazione gli strumenti giuridici di decretazione personale con l'operato dei molti "piccoli Führer" che operarono in una entità amministrativa atipica come il Governatorato generale di Polonia mostrando, talvolta prima con l'inadeguatezza che con la brutalità, il volto peggiore della Zivilverwaltung.

Parole chiave: Führererlasse; Zivilverwaltung; Kreise; Hans Frank; Kreishauptmann; Stadthauptmann.

Sommario: 1. Introduzione. - 2. Lo strumento di decretazione personale: i Führererlasse. - 3. Eccessi e incongruenze nell'amministrazione locale: I'impatto col GG. - 4. Una strana forma di "ordinaria amministrazione": violenze e altre illegalità. - 5. Quando il "diritto" è allo sbando. -6 . Conclusioni. 


\section{Introduzione}

Il sistema basato sulla dottrina politica nazionalsocialista non nacque accompagnato né da una base culturale giuridica propria, né sul presupposto di "appoggiarsi" allo Stato per poter riorganizzare la vita sociale ed economica tedesca. In effetti, furono i numerosi giuristi nazionalsocialisti ad elaborare una sorta di "struttura delle fonti giuridiche" che costituisse una riserva di strumenti giuridici da utilizzare per raggiungere un punto di "fusione" tra impianto istituzionale e dottrina politica di indirizzo.

Gli effetti distorsivi che il nuovo "Diritto Nazionalsocialista" ebbe nella gestione dei territori, specie in quelli occupati durante la guerra, si manifestarono con maggiore intensità sul territorio polacco, o meglio su quella porzione di territorio rinominato Generalgouvernement für die besetzten polnischen Gebiete, o più semplicemente Governatorato generale di Polonia ${ }^{1}$; sia negli alti livelli della Zivilverwaltung (amministrazione civile), sia nell'amministrazione dei distretti locali (Kreise), i personalismi dei funzionari si manifestarono con forza, anche grazie all'uso di forme di decretazione personale dotate di differente efficacia. L'obiettivo della "codificazione" del regime divenne quindi oggetto di strumentalizzazione, sia da parte delle alte sfere del partito (Hitler compreso), sia da parte di coloro che operarono nell'amministrazione del GG, specialmente di alcuni funzionari locali che, in forme differenti e con diversa intensità, esercitarono le loro funzioni come dei piccoli dittatori, utilizzando versioni della decretazione personale giuridicamente differenti e limitate territorialmente.

Dopo l'invasione della Polonia, gli individui assoggettati che rimasero nelle zone amministrate dai tedeschi furono sottomessi ad una forma di Stato (-apparato e -ordinamento) particolare, il GG appunto, istituito per decreto il 12 ottobre 1939 ed operativo dal 26 ottobre dello stesso anno².

II Governatore Hans Frank ${ }^{3}$ si trovò di fatto nella posizione di dover giustificare di fronte al suo Führer la guida del «Gau dei Vandali» e il suo essere al contempo un uomo di legge. Hitler esternò il proprio disprezzo verso la categoria dei giuristi che considerava "traditori del popolo», definendoli «minorati mentali dalla nascita o destinati a diventarlo con il passare del tempo ${ }^{4}$ e dichiarò in un discorso al Reichstag del 26 aprile 1942 che «non si sarebbe dato pace finché ogni tedesco non si fosse convinto che essere giuristi era una vergognosa ignominia» ${ }^{5}$. Curiosamente, l'effettivo esercizio del potere da parte del dittatore, impostato sulla

\footnotetext{
${ }^{1}$ In seguito $\mathrm{GG}$.

${ }^{2}$ Secondo Hitler, il Governatorato non sarebbe dovuto essere né una parte del Reich tedesco, né un distretto amministrativo del Reich.

3 Ex avvocato personale di Hitler, membro fondatore del Nationalsozialistische Rechtswahrerbund, o NSRB (Associazione dei giuristi nazionalsocialisti), e a capo della Akademie für Deutsches Recht (Accademia del diritto tedesco).

${ }^{4}$ Ritter (ed.), 1952, p. 225.

${ }^{5}$ Frank, 1953, p. 116.
} 
sua indiscussa autorità personale e politica, venne giustificato sul piano giuridico dall'interpretazione dottrinale di alcuni singolari strumenti normativi, i Führererlasse, la massima espressione di "compromesso", tra giustificazione del potere esistente e tentativo di codificarne la forza e i contenuti in una peculiare struttura "costituzionale" nazista.

Essi non fecero mai parte formalmente dell'ordinamento tedesco, ma furono de facto un elemento approvato e ampiamente giustificato dalla dottrina giuridica nazionalsocialista, che andò ad influenzare la vita del Reich e dei territori da esso controllati, alimentando ed amplificando quel sistema di doppi livelli istituzionali che ebbe nel GG la più caotica delle realizzazioni.

\section{Lo strumento di decretazione personale: $i$ Führererlasse}

La divisione della quotidianità tra tedeschi e slavi fu realizzata attraverso un piano normativo basato su una "fonte del diritto" di tipo "superprimario", ossia il decreto personale. Nel quadro normativo nazionalsocialista, per decreto personale si intendeva innanzitutto (o, per meglio dire, unicamente) il Führererlass ma, durante gli anni della guerra, l'ulteriore personalizzazione di questo strumento assunse i caratteri della "settorialità" e della "territorialità": il primo riguardò principalmente il settore della sicurezza e la polizia ed ebbe come soggetto promulgatore il Reichsführer delle SS Himmler; nel secondo caso invece, il GG offrì un fertile terreno istituzionale per l'affermazione dell'autorità politica e giuridica dei funzionari della Zivilverwaltung, da Frank in giù.

Considerando la superiorità gerarchica dei provvedimenti e degli atti normativi collegati alla carica di Hitler, ossia ordine-comando (Befehl), decreto (Erlass), regolamento (Verordnung), ordine-disposizione (Anordnung) e ordinanza (Verfügung $)^{6}$, mi sembra opportuno fare alcune osservazioni per determinarne la natura e la funzione, considerando che questi atti legislativi costituirono una novità nella storia "costituzionale" tedesca. I pronunciamenti di Hitler, tralasciando il ruolo di comandante in capo della Wehrmacht, ricordano quelli di un monarca assoluto. Possono essere dunque facilmente inquadrabili come strumenti, di vario livello ed efficacia, caratterizzanti l'aspetto giuridico della dittatura hitleriana, sia se utilizzati direttamente dal Führer, sia se "presi a prestito" da molti funzionari di primo piano della nomenclatura nazista e impiegati, in riferimento al livello e alla "forza giuridica" necessari, per conferire autorevolezza ed efficacia immediata ai singoli provvedimenti, tanto "settoriali" (ossia nei rapporti tra funzionari amministrativi, di pari o differente livello, civili e/o di polizia) quanto "territorali" (attuati negli Ehemalige Deutsche Ostgebiete)7.

\footnotetext{
${ }^{6}$ Queste cinque categorie rappresentarono circa il 90\% di tutte le direttive di Hitler, mentre gli atti collegiali del governo del Reich furono il 10\% circa; in Moll (ed.), 2011, p. 17.

${ }^{7}$ Per un quadro generale, cfr.: Majer, 1987; Böckenförde (ed.), 1985; Echterhölter, 1970.
} 
Fino all'estate del 1934, non vi fu traccia delle ordinanze e dei decreti personali di Hitler tra le pagine della Reichsgesetzblatt ${ }^{8}$. Vi erano solamente regolamenti e circolari dei singoli ministri, oppure leggi della Reichsregierung nelle quali Hitler è citato come "Cancelliere del Reich». Nel primo anno e mezzo di governo, le sporadiche decretazioni "di tipo personale" prodotte da Hitler, come il decreto sulle funzioni e sui compiti del Reichsministeriums für Wissenschaft, Erziehung und Volksbindung (Ministero della Scienza, dell'Istruzione e dell'Educazione Nazionale) dell'11 maggio 1934, non configurano la persona del Führer come vero e proprio legislatore; egli agiva sempre in un quadro di raccordo con il Presidente del Reich, quasi come un organo esecutivo9.

Manca inoltre la (successiva) tipica intestazione di «Decreto del Führer e Cancelliere del Reich». Dopo un dibattito e una sorta di "conflitto di attribuzioni" con il governo, Hitler emanò un ordine il 27 luglio 1934 con il quale il dittatore, pochi giorni prima della morte di Hindenburg e senza alcun ricorso ad una legge di delega, dispose la partecipazione di Rudolf Hess, vice di Hitler e ministro del Reich senza portafoglio ("Stellvertreter des Führers» und Reichsminister ohne Geschäftsbereich) alla preparazione di tutti i disegni di legge (Gesetzesentwürfe) nella posizione di «ministro del Reich interessato $»^{10}$.

II 2 agosto 1934, alla morte di Hindenburg, venne infatti emanato il «Decreto del Presidente del Reich per l'attuazione della legge sul capo di stato del Reich tedesco del $1^{\circ}$ agosto 1934$)^{11}$. Si possono individuare alcuni elementi caratterizzanti questa nuova forma di decretazione: I'intestazione "Erlass des Reichskanzlers» (decreto - personale - del Cancelliere del Reich), l'assenza di un riferimento a qualunque base normativa che autorizzasse o limitasse tale tipologia di decretazione, così come una terminologia che denotava un tono di sicurezza della propria posizione e un personalismo "insolito" per un testo di natura giuridica (tra le espressioni adoperate, "lo voglio che...» e «lo esigo che...»)"

Lo status "costituzionale" del Führer non era giustificato da uno stato emergenziale, ma dalla volontà di accentramento totale dell'autorità politica. Ne furono un esempio altri tre Führererlasse datati 2 agosto 1934 riguardanti: l'istituzione del Reichs-Justizprüfungsamt (l'ufficio del Reich per il controllo sulla giustizia), una disciplina in materia di insediamenti e alloggi, nonché il diritto alla grazia

\footnotetext{
${ }^{8}$ RGBI., 1934, I, s. 375.

${ }^{9}$ Moll, 2011, p. 17.

${ }^{10}$ Copia dell'ordine emesso su carta intestata del Reichskanzler, in BAB, R 43, II/141, p. 12. Lettera accompagnatoria di Lammers al Reichsminister del 27 luglio 1934, in Moll, 2011, p. 11, in cui si parla di un "Erlass".

${ }^{11}$ Legge sul capo di stato del Reich tedesco del $1^{\circ}$ agosto 1934, RGBI., 1934, I, p. 747. Decreto del Cancelliere per l'attuazione della legge sul capo di stato del Reich tedesco del $1^{\circ}$ agosto 1934, operativa dal 2 agosto 1934. Moll, 2011, p. 751. Questo decreto venne stranamente inteso come un messaggio al Ministro degli Interni e inizia con le parole «Signor Ministro degli Interni!» (Herr Reichsinnenminister!).

12 Moll, 2011, p. 18.
} 
nelle cause penali ${ }^{13}$. Essi costituirono indirettamente il punto di svolta sostanziale del potere hitleriano, conferendo al dittatore la giustificazione necessaria per il ricorso costante alla decretazione personale ${ }^{14}$. Peter Hubert pose l'interessante quesito sul perché, fino al 1945, non fu concesso per legge il monopolio del potere legislativo al dittatore, sebbene nei primi anni di regime la giurisprudenza nazionalsocialista si fosse impegnata nel configurare una pur discutibile tutela giuridica al suo operato e allo strumento della decretazione personale ${ }^{15}$. Per ragioni che peraltro lo stesso Hubert non riesce a dettagliare sul piano tecnico, non vi fu una puntuale codificazione di tipo costituzionale basata sul Führerprinzip, poiché il Reichstag mantenne il potere legislativo per tutti i dodici anni di regime ${ }^{16}$.

Anche la cosiddetta legge sulla ricostruzione (Neuaufbaugesetz) del $1^{\circ}$ gennaio 1934, deliberata dal Reichstag, autorizzò la produzione normativa del governo del Reich «in quanto organo collegiale», senza indicare il Führer o un singolo ministro ${ }^{17}$. È interessante notare che, poco dopo lo scoppio della guerra, Hitler si espresse sull'opportunità di riservare alcuni compiti legislativi al governo del Reich: «In ogni caso, le norme giuridiche, che per la loro importanza devono recare la firma del Führer, dovrebbero essere varate dal governo del Reich sotto forma di legge del Reich ${ }^{18}$. Tuttavia, nel corso dei mesi questa linea di indirizzo fu applicata sempre meno, a partire dalle prime settimane successive alle fine della Campagna di Polonia.

La situazione giuridico-costituzionale, all'inizio della guerra, era solo formalmente caratterizzata da una certa "debolezza" del Führer, che sul piano del diritto vigente non aveva alcuna potestà legislativa, né esclusiva né concorrente (con il Reichstag, il governo e il popolo, attraverso i referendum). Hitler non si lasciò confinare nel ruolo previsto, più o meno direttamente, dal diritto, e non rinunciò alla "libertà d'azione", "guadagnata" dopo la morte di Hindenburg.

Durante la guerra vi fu il costante ricorso ai Führererlasse; si assistette contestualmente al declino del decreto del governo come "strumento giuridico alternativo" ${ }^{19}$. I decreti personali di Hitler rappresentarono dunque il perno giuridico e operativo su cui il regime nazionalsocialista costitui il funzionamento del sistema

\footnotetext{
${ }^{13}$ RGBI., 1934, I, pp. 845, 1069, 1225.

${ }^{14}$ Per un quadro generale, cfr. Hubert, 1992.

${ }^{15}$ Hubert, 1992, p. 16.

${ }^{16}$ Hubert, 1992, p. 60.

17 RGBI., 1934, I, p. 75. Anche la cosiddetta legge delega (Ermächtigungsgesetz) del 24 marzo 1933 (RGBI., 1933, I, p. 141), prorogata poi nel 1937, 1939, 1943, standardizzò i poteri del governo come organo collegiale. Con riferimento all'ultima proroga della legge, del 10 maggio 1943 (RGBI., 1943, I, p. 295), cfr. le considerazioni di Hubert in Hubert, 1992, pp. 140-2; 205-6.

${ }^{18}$ Circolare di Lammers ai membri del Consiglio di Difesa del Reich e ai Ministri del Reich del 20 Febbraio 1940, in BA R 43 II/1648, p. 146; viene riportato nel documento il pronunciamento di Hitler sul tema.

${ }^{19}$ Per un riferimento generale, cfr. Schaefer A., 1985, pp. 89-105.
} 
statuale, sia all'interno del Reich sia nei territori occupati, in particolare il GG, mediante un sistema di legislazione eccezionale permanente ${ }^{20}$.

Non mancarono tuttavia i tentativi di noti giuristi del regime per armonizzare questa discrepanza giuridica.

Le incongruenze formali del sistema giuridico nazionalsocialista furono oggetto di studio da alcuni giuristi di regime, riscontrabile in alcuni articoli di riviste tedesche specializzate degli anni Trenta e Quaranta ${ }^{21}$. A partire dagli anni Novanta, lo studio del diritto durante il nazismo ha registrato una serie di fondamentali contributi, come nel caso di Michael Stolleis; per questo motivo, è necessario sottolineare la singolarità del quadro italiano, caratterizzato da una sostanziale indifferenza verso questi testi da parte della quasi totalità degli storici.

I cambiamenti nella prassi costituzionale e amministrativa del Reich furono accuratamente registrati e analizzati dalla dottrina, sebbene non siano stati interpretati come segnali involutivi di disintegrazione del sistema costituzionale, ma come una forma di consolidamento della leadership hitleriana. L'adozione di strumenti giuridici come il disegno di legge e la bozza di regolamento (nell'ambito di una sorta di "tecnica di rotazione degli atti normativi"), attraverso i quali Hans Lammers ${ }^{22}$, nei primi due anni circa di guerra, assurse al livello di "Ministro della Legge", anziché delle consuete precedenti deliberazioni all'interno del Gabinetto del Reich (presieduto da un "distratto" Hitler), trovò alcuni attenti osservatori. A fronte di un'autentica frammentazione degli uffici amministrativi e di governo del Reich (Huber menziona un totale di 42 uffici governativi, di cui 38 all'interno del territorio tedesco e 4 nei territori occupati, tra cui lo stesso $G^{23}$ ) e la conseguente moltiplicazione degli atti normativi emanati dalle varie autorità, il decreto personale del Führer rappresentò il fulcro giuridico attorno al quale gravitarono tutti gli altri atti giuridici; «l'unità dell'amministrazione per la guida del Reich» e la tenuta politica del paese in guerra, furono strutturate sulla centrale figura di Hitler ${ }^{24}$. La conseguente parziale riattivazione degli organi collegiali governativi consentì di "alleggerire" il peso dei lavori del Consiglio dei ministri per la difesa del Reich (Ministerrat für die Reichsverteidigung), piuttosto attivo all'inizio della guerra ${ }^{25}$. Nonostante l'ampia attività del Consiglio, Hitler mantenne uno stretto controllo su tutte le funzioni di governo; perciò Ernst Rudolf Huber, luminare del diritto costituzionale nazista, sostenne l'inesistenza del pericolo di

\footnotetext{
${ }^{20}$ Hubert, 1992, p. 61.

${ }^{21} \mathrm{Si}$ cita come esempio il dibattito sulla inedita decretazione di Hitler in materia di rafforzamento della germanicità del 7 ottobre 1939, in Huber, 1941, pp. 530-79 (qui p. 560).

${ }^{22}$ Capo della Cancelleria del Reich dal 30 gennaio 1933 al 24 aprile 1945.

${ }^{23}$ Huber, 1941, p. 561.

${ }^{24}$ Huber, 1941, p. 561. Osservazioni sul crescente numero e sull'importanza dei cosiddetti Führerakte si trovano anche in Weber, 1942, pp. 101-37 (qui p. 101).

${ }^{25} \mathrm{Cfr}$. inoltre i progetti per un decreto di Hitler «sul suo temporaneo alleggerimento da questioni di governo e amministrative in» in BA R 43 II/958, pp. 32-3.
} 
separazione tra governo e leadership dello stato, in ottemperanza ai dettami del Führerprinzip ${ }^{26}$.

Data l'incoerenza formale degli atti di Hitler rispetto al sistema giuridico vigente e l'impossibilità di mascherarla da parte della dottrina nazionalsocialista attraverso semplici circonlocuzioni ${ }^{27}$, Huber e altri studiosi decisero di inserire apertamente nel panorama della giurisprudenza "costituzionale" tedesca delle formule giuridiche che accentuassero il carattere preminente della concentrazione del potere nella figura del Führer e nella personalità espressa attraverso la decretazione personale nel quadro del Führerstaat ${ }^{28}$.

Dal momento che Hitler rappresentava l'unica carica suprema col potere di decidere sulle questioni di pace o guerra, il dibattito tra giuristi sulla natura e sull'origine degli atti normativi diretti del Führer e sulla possibilità di considerarli autonomi o derivativi risultò irrilevante. Tanto più che le varianti tecniche dei decreti personali mantenevano l'elemento essenziale, ossia l'esercizio diretto della volontà del Führer, indipendente e strutturalmente superiore a qualunque strumento giuridico. Venne addirittura negata, da parte del Dott. Werner Best, avvocato e vice di Reinhard Heydrich presso il quartier generale della Gestapo, ogni distinzione tra norme forti e deboli, tra legge costituzionale e legge ordinaria, tra leggi, ordinanze e decreti, tra diritto pubblico e privato, poiché: «La volontà da parte della guida politica, in qualunque forma essa trovi espressione, crea la legge e modifica la legge esistente ${ }^{29}$.

La dottrina sostenne sempre l'assoluta onnipotenza di Hitler sul piano giuridico e la sua insindacabile facoltà di adottare decisioni attraverso leggi, decreti o qualunque altro atto normativo. Huber riassunse così il principio del Führermacht: «ll potere del Führer è esclusivo, onnicomprensivo e illimitato; esso è sovrano» ${ }^{30}$; ciò ha portato alla parificazione delle tipologie di atti adottate dal Führer. Secondo questo principio, come riportato da Werner Best, «dal momento che la legge, così come l'ordinanza governativa, l'ordinanza amministrativa, l'ordine di servizio, la disposizione unica [costituiscono] in definitiva un Führerbefehl (ordine del Führer), non è possibile attribuire alla legge alcuna priorità ${ }^{31}$. Ogni parere critico dei giuristi a questo orientamento esistente negli anni Trenta scomparve durante

\footnotetext{
${ }^{26}$ Huber, 1941, p. 553.

${ }^{27}$ Cfr. Korte, 1942, pp. 473-6, 498-501 (qui pp. 499-501); Weber, 1942, pp. 123-7. Nel caso, la decretazione personale venne presentata come un dogma, in cui i singoli atti presentavano spesso una singolare formulazione, indicata come "buntes Bild" (immagine variopinta), alla quale non era sempre attribuibile un significato squisitamente giuridico.

${ }^{28}$ Korte, 1942, p. 498.

${ }^{29}$ Best, 1941, pp. 15-6. L'autore afferma come l'azione statale sia l'affermazione della volontà di un leader «attraveso un singolo ordine o la determinazione e accettazione di regole che siano giuridicamente vincolanti indipendentemente dalla forma».

${ }^{30}$ Huber, 1935, pp. 202-29 (qui p. 228).

${ }^{31}$ Cfr. Best, 1938, pp. 44-50; cit. in Herbert, 1996, p. 179.
} 
la guerra ${ }^{32}$.

A partire dal 1943 il tema della maggiore efficacia della legge rispetto agli altri atti normativi emanati da Hitler cessò di suscitare interesse per i giuristi tedeschi e fu considerata una "ovvietà" sul piano giuridico ${ }^{33}$.

In merito al dibattito storico sulla effettiva centralità di Hitler nel regime nazista, l'interpretazione della natura dei Führererlasse e il loro inserimento nel quadro "costituzionale" dello stato possono essere d'aiuto nel definirne i parametri ${ }^{34}$.

La costruzione di una visione d'insieme sulla natura giuridica delle decisioni prese da Hitler è sicuramente molto difficile, così come la conseguente analisi del grado di esclusività che avrebbero dovuto avere i decreti personali; non è infatti possibile affidarsi solamente alle pubblicazioni sul Reichsgesetzblatt (Gazzetta del Reich).

Di conseguenza, la proliferazione della decretazione personale nei territori polacchi occupati, con riferimento all'amministrazione civile e alle SS, può essere generalmente intesa sotto due profili: il primo, di natura personalistica, garanti a ciascun funzionario la possibilità di affermare la propria autorità politica, tanto verso i propri subordinati quanto verso i colleghi di pari livello e nei confronti delle popolazioni assoggettate; la "straordinarietà" delle condizioni li rese titolari di un potere quasi illimitato, esercitato in un contesto di tipo quasi "feudale". ॥ secondo, di carattere "tecnico", ebbe la indiretta conseguenza di rafforzare uno strumento giuridico che accrebbe l'efficacia dell'azione amministrativa sul territorio.

Al fine di determinare le ragioni di un esercizio così personalistico del potere, cui furono così funzionali questi strumenti giuridici, sembra dunque opportuno valutare il contesto territoriale, l'impatto col nuovo "ambiente di lavoro" dei funzionari chiamati nel GG, il processo di "integrazione" che li accompagnò nell'opera di gestione, ma soprattutto il peso delle tematiche razziali nei distretti.

Per indicare le dinamiche interne alle amministrazioni locali, credo risulti opportuna una presentazione di alcuni casi significativi, che meglio qualificano il rapporto tra soggetti, sistema giuridico e territorio.

\section{Eccessi e incongruenze nell'amministrazione locale: l'impatto col GG}

L'analisi del lavoro degli amministratori locali non può prescindere da un approccio di tipo "istituzionale", fondamentale per cercare di comprendere i rapporti sia

\footnotetext{
${ }^{32}$ II noto costituzionalista di regime Otto Koellreutter si espresse così su questo argomento: "Questi modelli, come la legge e l'ordinanza, hanno ciascuno le proprie peculiarità. Pertanto nello stato nazionalsocialista non tutte le espressioni della Führung agiscono come una legge. Per essere efficace come una legge, il Führerwille dovrà vestire i panni giuridici della legge». Koellreutter, 1938, p. 15.

${ }^{33}$ Cfr. a riguardo Rüthers, 1988.

${ }^{34}$ Zitelmann, 1992, pp. 491-506.
} 
tra i diversi livelli di amministrazione sia al loro interno. L'architettura istituzionale del GG garantiva, più per inadeguatezza che per formazione, una certa significativa libertà d'azione ai singoli capi-area, le cui efficienza e credibilità dipendevano dalle personalità dei singoli Kreishauptmänner (capi dei distretti locali) e Stadthauptmänner (capi dei distretti cittadini).

Rispetto all'esercizio delle attività di occupazione, si tratta di comprendere la predisposizione degli amministratori tedeschi nel prestarsi automaticamente a processi di radicalizzazione nel trattamento delle popolazioni dominate, come polacchi ed ebrei, con le distinzioni derivanti dalla dottrina razziale. Inoltre va intesa la portata dei margini di manovra e della discrezionalità di cui essi godevano, una libertà d'azione, tacitamente concessa e autogestita, le cui conseguenze furono incompatibili con un esercizio equilibrato dell'attività amministrativa all'interno della peculiare forma istituzionale del GG.

Quanto contarono le motivazioni ideologiche? Quanto la predisposizione alla violenza dei singoli soggetti e il carrierismo privo di scrupoli diffuso nella burocrazia nazista? Fino a che punto la radicalizzazione degli occupanti trasse origine dall'interno della macchina amministrativa?

Con la destituzione di molte delle autorità politiche e istituzionali locali, il paese ha perso parte di quelle figure che mantenevano il legame (peraltro flebile) tra le diverse identità che componevano la società polacca, separandone di fatto alcuni settori e isolando sia la classe degli intellettuali polacchi sia la comunità ebraica. Questa "atomizzazione delle relazioni sociali" venne accelerata dall'operato degli occupanti con una brutalità senza precedenti e con la gerarchizzazione, su base etnica, del modello amministrativo e sociale nazionalsocialista ${ }^{35}$.

Il corpo eterogeneo degli amministratori locali tedeschi del GG rivelò spesso gli aspetti più duri del dominio tedesco nei confronti dei polacchi, disprezzati e umiliati durante la gestione della quotidianità ${ }^{36}$.

«Sterile, vuota, desolata, una stazione in macerie, sporcizia, puzza, gentaglia, una lingua straniera, a malapena una parola di tedesco, [questa] è stata la prima accoglienza ${ }^{37}$; questo fu il primo impatto di Goebbels alla vista di Cracovia. Heinz Doering, giurista bavarese in servizio presso il distretto di Mühldorf, trascorse solo un paio di giorni nella capitale del Governatorato generale, come riportato nelle lettere alla madre dell'aprile del 1942. Con riluttanza seguì la moglie nel GG, mostrandosi disgustato da «tutta questa gentaglia orientale semiumana (Halbmenschentums)1» ${ }^{38}$.

\footnotetext{
${ }_{35}$ Młynarczyk, 2004, pp. 145-6.

${ }^{36}$ Riferimenti in Harten, 1996, pp. 86-7.

${ }^{37}$ Lettera di Heinz Doering alla madre del 28 aprile 1942, in Roth, 2009, p. 7. Riferimenti più ampi sulla figura di Doering, principalmente riguardo il ruolo di giurista e amministratore, in Friedrich, Heim, 2013, pp. 274 segg.

${ }^{38}$ La documentazione sull'afflusso di personale, civile e SS, dal Reich alla Polonia occupata, e sui successivi smistamenti all'interno del GG, è vasta e frastagliata. Riferimenti sul tema sono presenti in molti archivi, tra cui Bundesarchiv Berlin (BAB) e Instytut Pamięci
} 
Il disagio di molti funzionari tedeschi "colpiti" da trasferimento si spiegava con la pessima reputazione di cui godeva il GG: il condizionamento prodotto dalla propaganda di Goebbels era talmente forte che quasi ogni funzionario rimase inizialmente traumatizzato dall'impatto col nuovo ambiente di lavoro, con le abitudini e con lo stato dei servizi, degli alloggi e delle infrastrutture. «ll buco era nero di sudiciume e puzzava. L'intonaco si staccava, il lavandino era rotto, i letti di ferro sembravano essere [...] rottam[i] [recuperati in] alcune baracche, i materassi avevano macchie di merda [vecchie di] generazioni» ${ }^{39}$; e ancora: «inganna la servile [...] gentilezza, con la quale i polacchi si mostrano di facciata. Alle spalle sono [invece] pericolosi» ${ }^{40}$.

Dopo i primi giorni di disperazione, i residui propagandistici e il trauma del trasferimento lasciarono spazio a più ottimistiche considerazioni sulle effettive condizioni riservate ai funzionari amministrativi tedeschi in terra polacca. Nello specifico caso di Doering, possiamo parlare di uno dei (non molti) casi di spostamento fondato sulla effettiva esigenza della Zentralregierung (governo centrale) di inserire personale competente "ariano" nel quadro di comando delle amministrazioni locali, che vedevano una componente polacca largamente maggioritaria. Dopo alcuni giorni di soggiorno a Cracovia, Doering scrisse alla madre: «Non lasciarti sfuggire nulla! Siamo così abbondantemente forniti di tutto [...] [da sentirci] come in paradiso e certamente non abbiamo bisogno di nulla». In effetti i benefits dei coniugi Doering furono molti e di varia natura: ricevettero «un appartamento, mobili, biancheria e molto altro, godevano di un buon [trattamento economico]» dal quale trassero un alto tenore di vita e si trovarono in condizione di «accumulare ricchezza» ${ }^{41}$.

Questo risvolto vantaggioso accrebbe lo spirito di iniziativa e le intenzioni di dominio di molti funzionari: "prima di tutto», asserì Doering, "non siamo qui per divertimento, ma come coloni! $»^{42}$. Ritorna dunque la considerazione del territorio del Governatorato come di una "terra delle opportunità" per i nazisti, da conquistare tramite lo sfruttamento intensivo delle risorse umane e materiali che il nuovo stato metteva a disposizione degli occupanti ${ }^{43}$.

Narodowej (Istituto di Memoria Nazionale - IPN). In particolare si ricorda l'iter seguito da Karl Heinrich Wilhelm Koppe nella sua carriera dalla Germania a Posen (maggio 1940 giugno 1941), poi tra Posen e Chelmno (giugno 1941 - 8 novembre 1943) e poi a Cracovia (9 novembre 1943 - 9 dicembre 1944). Circolare indirizzata al Procuratore Capo presso la corte distrettuale di Bonn, al secondo tribunale penale della corte distrettuale di Bonn, atto d'accusa, Bonn, 10 settembre 1961, in IPN, BU-2586-329, pp. 4-6. La circolare fa riferimento all'arresto di Koppe, avvenuto nel 1960 a Bonn, dove l'ex gerarca viveva con lo pseudonimo di Wilhelm Karl Heinrich Lohmann, avendo assunto il cognome della moglie.

${ }^{39}$ Lettera di H. Doering alla madre del 28 aprile 1942, Roth, 2009, p. 7.

${ }^{40}$ Lettera di H. Doering alla madre del 5 maggio 1942, Roth, 2009, p. 8.

${ }^{41}$ Lettera di H. Doering alla madre del 5 maggio 1942, Roth, 2009, p. 8.

${ }^{42}$ Roth, 2009, p. 8.

${ }^{43}$ Riferimenti in Schwaneberg, 2009, pp. 133-54, https://ipn.gov.pl/pl/szukaj?sear- 
Il percorso professionale del giurista bavarese può essere considerato un esempio di piccolo carrierismo tipico dei funzionari locali: promosso a capo reparto dopo sole due settimane di permanenza nel Distrikt Krakau, fu progressivamente spostato verso i centri periferici del distretto fino alla realizzazione del suo obiettivo più importante, ossia la nomina a Kreishauptmann. Dall'agosto 1943 all'agosto 1944 ricoprì infatti la carica di Stadthauptmann di Opatów ${ }^{44}$, nel distretto di Radom, in sostituzione del Landrat (presidente del distretto rurale) Heinz Ritter (in carica dal gennaio 1940) ${ }^{45}$.

Le condizioni di lavoro favorevoli agli amministratori tedeschi non furono sufficienti a nascondere la natura e le conseguenze dell'indottrinamento nazista nel rapporto col territorio occupato. L'ostilità e l'esercizio della violenza nei confronti (principalmente) di polacchi ed ebrei costituì una sorta di tacita conditio sine qua non per una "corretta" applicazione dei principi amministrativi del nazionalsocialismo.

«D'altra parte [dobbiamo], almeno in via provvisoria, rinunciare alla politica dei trasferimenti e di estinzione, perché non abbiamo abbastanza person[ale] e quindi per il momento [c'è] ancora bisogno de[lla manodopera] polacc[a] [...]. Chi [...] altrimenti, per esempio, [potrebbe] lavor[are] nei campi? $»^{46}$. L'insieme di pragmatismo e radicalismo nell'approccio degli amministratori tedeschi alla loro "nuova missione" rivelò tutto il condizionamento ideologico, che non lasciava spazio alla pietà o alla compassione. Al contrario: la dottrina li aiutò a respingere questi impulsi, giustificandosi attraverso un'autodifesa razionalmente costruita.

Oltre al disprezzo, vi fu lo scherno e la repulsione per le pessime condizioni della popolazione, che venivano imputate agli stessi polacchi, visti come colpevoli della loro scarsa qualità di vita. Con gli ebrei però l'accanimento fu più marcato: inquadrati come un "non essere", non vi era alcuna limitazione, neppure formale, agli attacchi perpetrabili contro di essi. Le operazioni di sterminio vennero anzi ricondotte all'interno di "parametri legali": gli amministratori tedeschi non furono, in linea generale, solamente gli esecutori materiali di circolari e direttive provenienti dall'alto, ma parteciparono ampiamente alla progettazione di alcuni provvedimenti, personalizzandone talvolta gli aspetti relativi alla situazione dei singoli Kreise.

Persone come Ernst Gram $\beta^{47}$, funzionario e membro del partito trasferitosi con

$\mathrm{ch}=4061521 \&$ sort=2\&order=1\&ile=20 (20-05-2021), documento caricato sull'archivio online dell'IPN il 16 aprile 2016.

${ }^{44}$ Jehke (ed.), Territoriale Veränderungen in Deutschland und deutsch verwalteten Gebieten 1874-1945, in http://www.territorial.de/gg/opatow/kreish.htm (20-05-2021), caricato il 20 agosto 2009.

${ }^{45}$ du Prel M. F., 1940, Das deutsche Generalgouvernement Polen: ein Überblick über Gebiet, Gestaltung und Geschichte, Krakau, BVO, p. 101, 133-4.

${ }^{46}$ Lettera di H. Doering alla madre del 5 maggio 1942, Roth, 2009, p. 9.

47 Riferimenti biografici su Gramß (o Gramss) in http://jewishsokolow.blogspot. 
la moglie dalla Germania nel novembre $1939^{48}$, vissero la ricollocazione nel GG come una sorta di shock culturale. Sua moglie descrisse disgustata il viaggio in treno verso Cracovia: «il primo impatto era di grande povertà, un quadro desolante, ma [apparentemente] non per gli effetti della guerra [...] nei singoli villaggi ebrei (Judendörfer) un'immagine semplicemente indescrivibile [...] la sporcizia [...] i tipi di

ebrei da coprirsi il volto alla sola vista, in parte deformati da nanismo, [dal] l'obesità ${ }^{49}$.

Presto Gramß trasse conclusioni radicali. Da Varsavia scrisse a sua moglie: «il quartiere ebraico è una vergogna, 300.000 ebrei [...] spettacoli criminali in abbondanza [...] estirpar[li] sarebbe un vantaggio per l'umanità ${ }^{50}$. Poco tempo dopo si ebbe il primo ebreo impiccato: le accuse più comuni prese a pretesto dai nazisti erano di alterare i prezzi e fare affari sul mercato nero; peraltro si trattava dello stesso tipo di affari da cui i funzionari tedeschi locali e molti soldati della polizia di sicurezza traevano grandi guadagni personali $i^{51}$.

Gramß non era un caso isolato; numerosi tedeschi chiamati a lavorare in PoIonia rimasero "scioccati". Un caporale descrisse le proprie impressioni in una lettera del 11 settembre 1940: "giunti a destinazione, ci trovammo in una città la cui popolazione era per l' $80 \%$ ebrea. Anche solo uno di loro [...] è uno spettacolo disgustoso, ma migliaia di questo ceppo sono eccessivi. Quale aiuto si può dare, questa gente non vuole e non può migliorare $»^{52}$.

Come riportato prima da Robert Seidel e poi da Markus Roth, nel novembre 1939 Frank ricevette i Kreishauptleute (dirigenti dei distretti locali) e Stadthauptleute (dirigenti dei distretti cittadini) del Distretto di Radom per essere informato sul lavoro svolto nelle prime settimane di vita del GG.

Il primo a esporre i risultati dell'attività amministrativa fu Karl Glehn, anch'egli arrivato dal Reich: con superbia espose un resoconto estremamente "gonfiato" sul tipo di "lavoro pioneristico" svolto: «In principio il chaos. Tutti fuori dalle fabbriche a lavorare per strada, furti, contrabbando, truffe, terreno ideale per gli ebrei. Tutto [necessitava di] controll[o] [...] $)^{53}$.

it/2011/08/ernst-gramss.html (16-06-2021), archivio online dell'associazione culturale ebreo-polacca Jewish Sokol'ów Podlaski.

${ }^{48}$ Ernst Gramß ricoprì, dal 30 novembre 1939 al 6 maggio 1940, la carica di capo del dipartimento per le forniture di cibo e l'agricoltura nel distretto di Varsavia. In http:// jewishsokolow.blogspot.it/2011/08/ernst-gramss.html (23-05-2021).

${ }^{49}$ Stralcio di una lettera senza data di Gramß a sua moglie, risalente presumibilmente al novembre o al dicembre 1939, in BAL, B 162/Dok-Slg. Polen, Ordn. 344, p. 45.

${ }^{50}$ Lettera senza data di Gramß alla moglie del dicembre 1939, sugli inizi del suo periodo di lavoro a Varsavia, Roth, 2009, p. 46.

${ }^{51}$ Engelking, Leociak, 2009, pp. 384-9.

${ }^{52}$ Manoschek, 1995, p. 17.

${ }^{53}$ Verbale della riunione dei governatori distrettuali, dei sindaci e dei commissari cittadini del distretto di Radom, 25 novembre 1939, in BAL, B 162/Dok-Slg. Polen, Ordn. 344, p. 
Glehn discusse sulla necessità di attuare il processo di arianizzazione dei territori con un costante sfruttamento dei terreni agricoli e della popolazione locale a favore del Reich, per preparare il territorio polacco sul piano economico alla completa germanizzazione e normalizzazione del sistema produttivo. Una parte di questo programma prevedeva pene molto severe per i polacchi che facevano ricorso al mercato nero e a traffici illegali per occultare parte dei raccolti e trarne guadagni personali (esattamente come facevano da impuniti i funzionari tedeschi). Glehn concluse il suo intervento dicendo che, nel rispetto di quei presupposti, «il lavoro regala gioia» ${ }^{54}$.

Il raggio d'azione dei singoli funzionari locali era così ampio che Glehn diceva spesso con sarcasmo di potersi nominare egli stesso giudice ed eseguire condanne a morte senza conseguenza alcuna per la sua persona. Riferite a Frank, queste affermazioni non incontrarono opposizione da parte del Governatore, che invitò i suoi capi locali a governare col pugno di ferro per il bene del Reich, anziché esercitare con accuratezza la funzione di raccordo tra gli amministratori. Frank incoraggiava apertamente i funzionari locali a superare il concetto di stretta competenza e a ricorrere a deviazioni e scorciatoie per assolvere al compito principale: «Per come stanno le cose ora, questo rappresenta il primo tentativo di una nuova modalità di gestione dell'insediamento tedesco, che attraverso le esperienze si sta costruendo una nuova via [...] Sono convinto che l'utilizzo di altre teorizzazioni arrechi solo disturbo [all'amministratore]». Frank incoraggiò ad agire in funzione anti-polacca e anti-ebraica nelle politiche di occupazione, impedendo che un solo polacco conservasse la libertà di circolazione: «nessun polacco potrà mai più osare [mischiarsi] con dei signori tedeschi. A questo scopo è compito dell'amministrazione essere disposta a sopprimere ogni polacco proveniente dal Reich». II GG secondo Frank avrebbe dovuto svolgere la già citata funzione di "pattumiera sociale del Reich" senza subire diktat da Berlino e dalle SS che minassero la "autonomia" del nuovo stato e l'aura di "autorevolezza" che il Governatore credeva di possedere.

\section{Una strana forma di "ordinaria amministrazione": violenze e altre illegalità}

La condotta eccessivamente violenta all'interno del GG, tanto delle SS quanto dei membri della Zivilverwaltung, venne apertamente criticata da molti funzionari amministrativi. Tale presa di posizione non aveva (salvo rarissime eccezioni) fondamenti di tipo "umanitario": la tortura inflitta quasi per gioco venne ritenuta un elemento superfluo e dagli effetti controproducenti; era come se l'abuso della forza potesse sminuire la "funzione sociale" che i nazisti credevano di svolgere

447.

${ }^{54}$ BAL, B 162/Dok-Slg. Polen, Ordn. 344, pp. 449, 458. 
nell'opera di "purificazione dei territori dagli influssi del veleno ebraico"55. Un esempio è dato da una lettera scritta da Heinz Doering alla madre in relazione alla vicenda di un sorvegliante che mostrava, a suo dire, divertimento nel frustare alcuni ebrei: «Così non va bene. Con lo sterminio degli ebrei sono piuttosto d'accordo, ma questo sadismo è troppo. Contro la crudeltà da macello si sono fatte regole. Perciò si dovrebbe, a mio parere, eliminare gli ebrei, ma non torturarli per divertimento [...] lo sarei per la eliminazione totale» ${ }^{56}$.

I Kreishauptmänner godevano di un potere molto esteso, comprensivo di possibilità pressoché illimitate nel ricorrere a varie forme di illegalità. Quando Ernst Gramß fu messo a capo della contea di Sokołów nel giugno 1940 si senti al settimo cielo, non solo per l'avanzamento di carriera, ma perché prese coscienza di quali altre forme di abuso erano concesse ai funzionari: principalmente il furto, la corruzione e lo sfruttamento delle strutture appartenute alle istituzioni polacche o a ex-cittadini facoltosi. Univa dunque l'euforia da potere per la possibilità di gestire settori come economia, agricoltura, gestione del territorio, foreste, lavoro, trasporti, libera circolazione, polizia (in concorrenza con le SS) alla sostanziale vita da nababbo grazie a privilegi come cavalli per l'equitazione, un parco personale con piscina per il nuoto, la sauna, una schiera di servitori al suo servizio (bambinaie, cuoche, camerieri). Poco dopo la sua nomina scrisse alla moglie che il lavoro era così piacevole da volere «rimanere per sempre» ${ }^{57}$.

L'atteggiamento da sovrano assoluto di Gramß era tipico dei responsabili di molti distretti locali nel GG: anche da questi comportamenti infantili e dall'ostentazione di uno sfarzo pagato da altri si riconosceva il dilettantismo di una larga fetta del personale amministrativo $0^{58}$.

I funzionari tedeschi vennero così progressivamente attratti dal lavoro nel GG e nel Warthegau col miraggio di privilegi materiali. Doering era «convinto di [lavorare] nell'amministrazione» perché "stufo di fare l'avvocato»; si dichiarava soddisfatto e riteneva gratificante poter vivere con sua moglie nel territorio che lo vedeva Kreishauptmann, anche se l'incarico era meno remunerativo della sua attività di giurista. I "grandi progetti" che dichiarava di avere erano generati dalla consapevolezza di una missione, permeati dunque tanto dall'ideologia quanto dall'ambizione personale, la stessa che a suo dire gli impedì di godersi assieme alla moglie i soldi guadagnati da avvocato e di avere «una vita migliore [composta da] poco lavoro, molto tempo libero e tanti soldi» ${ }^{59}$.

\footnotetext{
${ }^{55}$ Ricorrente espressione utilizzata da Hitler per definire la funzione della Soluzione Finale.

${ }^{56}$ Lettera di H. Doering alla madre del 29 settembre 1942, in Roth, 2009, p. 49.

${ }^{57}$ Lettera di Gramß alla moglie, s.i.d., probabilmente risalente al 2 giugno 1940, in Roth, 2009, p. 50.

${ }^{58}$ BAL, B 162/II 211 AR-Z 73/66, parte 2, pp. 16, 140. Per riferimenti ai comportamenti dei funzionari vedi Madajczyk, 1987, p. 179.

${ }^{59}$ Lettera di H. Doering alla madre del 30 luglio 1942, in Roth, 2009, p. 52.
} 
Come riportato da Markus Roth, «non solo l'autonomia e la vita confortevole dei capi-distretto locali rese questa carica cosi appetita; vi si aggiunsero un errato idealismo e una fanatica e personale visione pioneristica del loro ruolo in Oriente ${ }^{60}$.

I capi-distretto e quelli cittadini si trovarono a ricoprire una funzione di governo del territorio orientale in "organismi [attivi] in una missione temporalmente limitata $»^{61}$. Nel nuovo Lebensraum im Osten, "serv[iva] comandare, non [...] governare». Joachim Nehring, nel suo articolo sulla Krakauer Zeitung, riportava: «Qui nulla è scritto. Qui valgono solo i fatti. Qui nessuno può dire non è affar mio poiché non rientra nelle proprie responsabilità. Ovunque ti trovi, sei nel Reich! In tutto ciò che accade, un polacco vede in te il Reich! Perciò non vi è che un limite alle vostre competenze e responsabilità: gli interessi del Reich. Energici pionieri, liberi da regole burocratiche [...], devono marcatamente ispirarsi [nel loro lavoro] in Oriente alle storiche origini tedesche ${ }^{62}$.

La prassi di comando si basava su una violenza consigliata e impunita: I'esistenza di alcune rarissime "oasi di normale amministrazione" dipendeva esclusivamente dalla volontà dei singoli capi locali e dalla risonanza che le loro azioni potessero avere ai piani alti della nomenclatura dello stato. La modalità di trattamento della popolazione era finalizzata a lasciare un segno nella politica di occupazione tedesca e mostrare la propria efficienza come amministratori attraverso lavori di costruzione (Aufbauarbeit) di una testimonianza della historische Mission nell'Ostgebiet. Hans-Adolf Asbach predispose il piano di ricostruzione e di organizzazione urbanistica della città di cui fu posto a capo, Brzeżany. Incaricò due architetti di Dresda di elaborare la nuova pianificazione urbana, sia per gli edifici pubblici sia per molte abitazioni private; il risultato avrebbe dovuto essere inteso in prospettiva storica: "Se oggi questi piani ci sembrano favolosi, posso solo dire che nulla è impossibile per i tedeschi ${ }^{63}$. Per i suoi piani, Asbach scelse come banco di prova un quartiere ebraico relativamente in buono stato, con case ben conservate; chiese al Judenrat un contributo per i lavori (Kontributionszahlung) in cambio del rilascio (temporaneo) di 600 ebrei. La truffa si realizzò crudelmente poco dopo il pagamento, quando i prigionieri rilasciati furono condotti qualche chilometro

${ }^{60}$ Discorso del 20 giugno 1943 di Viktor von Dewitz, Kreishauptmann di Stryj (Distrikt Galizien), in Diensttagebuch (DTB), IfZM, Fb 105/30, p. 7466.

${ }^{61}$ Nehring, 1940, Dienst im Osten, in „Krakauer Zeitung“, 11 giugno, n. 136; http://bc.radom.pl/dlibra/plain-content?id=23554 (26-05-2021). Archivio online della biblioteca cittadina di Radom.

${ }^{62}$ Nehring, 1940. Frank durante un incontro dei presidenti dei distretti locali del distretto di Cracovia dell' 8 novembre 1939, DTB, IfZ, Fb 105/1, BI. 40. La valutazione sul rapporto tra le opportunità concesse ai singoli funzionari di distretto e il "sacrificio" di dover risiedere in terra polacca accomunava molti capi distretto locali. Baedeker, 1945, passim. ${ }^{63}$ Hans-Adolf Asbach, "Von Krakau bis Brzeżany (1 Januar 1940 - 13 Februar 1943)», in Roth, 2009, p. 54. 
fuori dalla città e li giustiziati sul posto ${ }^{64}$.

Durante un discorso del 2 marzo 1940, Frank mostrò un certo "pragmatismo", lontano dai radicalismi di certe frange del regime nazionalsocialista: «Noi non possiamo, dopo tutto, uccidere quattordici milioni di polacchi! [...] [poiché] non disponiamo di person[ale sufficiente] per costituire un apparato [amministrativo]». Sei giorni dopo parlò della responsabilità del GG nel garantire un regolare svolgimento della vita quotidiana per tutti i «non-tedeschi» residenti nel territorio dello stato: "Come potrebbero [altrimenti] le altre nazioni desiderare di [finire] sotto la protezione tedesca?» ${ }^{65}$.

Al di là della condotta nei confronti delle popolazioni assoggettate, vi furono anche altre "abitudini" dei funzionari amministrativi tedeschi che finirono con l'essere giustificate dall'abuso della propria autorità, e della strumentazione giuridica a loro disposizione. La corruzione, l'arricchimento personale, i vizi, le abitudini sessuali, tutti elementi che smentivano la già peraltro discutibile difesa dei funzionari tedeschi da parte di Frank. Heinz Doering analizzò correttamente lo scenario, causato da una cattiva selezione e dalla diffusa incompetenza dei funzionari; indignato, scrisse a casa: «se penso a ciò che era una volta un ministero bavarese, le sue attività, l'ordine, la sua pulizia interna e la disciplina! E soprattutto le persone! [Oggi] sembra che la Germania abbia mandato tutta la feccia e la sporcizia qui, a far niente, ciascuno peggiore di dieci negri nudi. Quasi tutti coloro che sono qui fanno schifo e sono stati cacciati [dai rispettivi luoghi di lavoro] e spediti qui. Invece di colonizzare con decoro, si trattano i territori orientali come una specie di mucchio di spazzatura, sul quale poter scaricare lo sporco ${ }^{66}$. Tali lamentele si potevano riscontrare ad ogni livello dell'amministrazione, tanto che lo stesso Sicherheitsdienst ${ }^{67}$ (servizio di sicurezza) sottolineò, non senza una dose di sarcasmo: «[era] come se si cercassero soltanto corrotti nel Governatorato generale ${ }^{68}$.

Doering riconosceva che, oltre alla corruzione e all'incompetenza diffuse, una grossa responsabilità dell'andamento generale fosse imputabile alla debolezza della leadership di Frank, poiché il Governatore passava buona parte del suo tempo tra Berlino e la sua tenuta di Kressendorf (Krzeszowice), lasciando i suoi subordinati liberi di fare praticamente ciò che volevano. II capo del SD del distretto di Galizia criticò apertamente il comportamento di Frank, accusandolo di scarso impegno lavorativo e di non riconoscere alcun tipo di legame morale o istituzionale con lo Stato, poiché ogni funzionario corrotto poteva appoggiarsi al Governatore e alla sua famiglia ${ }^{69}$.

\footnotetext{
${ }^{64}$ BAL, B 162/202 AR-Z 76/61, parte 2, pp. 469, 506.

${ }^{65}$ Präg, Jacobmeyer, 1975, pp. 146-9.

${ }^{66}$ Präg, Jacobmeyer, 1975, pp. 146-9.

${ }^{67}$ In seguito SD.

${ }^{68}$ Lettera di H. Doering alla madre del 29 giugno 1942, in Roth, 2009, p. 59.

${ }^{69}$ Roth, 2009, p. 59. II giudizio del SD fu sfruttato da Krüger per screditare l'immagine di
} 
Uno dei casi più interessanti di contrasto fittizio alla corruzione, ma di fattiva condotta corruttiva da parte di funzionari locali fu quello di Karl Valentin, capo della contea di Ostrów, nel distretto di Varsavia. Giunto nella Polonia occupata da soldato dopo una militanza nelle SA, in poco tempo Valentin divenne collaboratore personale del governatore del distretto di Varsavia Ludwig Fischer ${ }^{70}$; il 19 gennaio 1942 fu nominato Kreishauptmann di Ostrów ${ }^{71}$.

Una volta iniziato a svolgere il proprio compito, si rese conto che il lascito dei suoi predecessori presentava problemi gravi: «I tempi sono così difficili che dobbiamo tenere un comportamento esemplare nella nostra attività e nella nostra vita privata quotidiana, non solo verso noi stessi, ma di fronte al popolo, alla patria, al Führer e, nel nostro specifico caso, di fronte ai polacchi. Finora non è stato così ${ }^{72}$. Valentin fece un appello per una maggiore cooperazione tra distretti locali, invitando i colleghi ad abbandonare la linea di isolamento dal governo centrale e biasimando l'ennesimo caso di arresto di un funzionario, in quel caso dell'Organizzazione Todt, accusato di frode, appropriazione indebita e furto.

Mi impegno ulteriormente per i miei compagni tedeschi e, in senso più ampio, anche per i laboriosi polacchi, poiché abbiamo bisogno di fare anche ciò che va oltre i nostri [rispettivi] ambiti. Non mi chiudo nei paragrafi dei libri di diritto. In nessuna maniera. Voglio sapere [come funzionano le cose nella realtà], in che modo io stesso potrei [cadere nella rete]. Al momento me ne occupo. Ho un piede e mezzo in un campo di concentramento. Ma me ne occupo ${ }^{73}$.

Gli ammonimenti e le accuse, oltre che i timori di ritorsioni nei suoi confronti, si rivelarono parole vuote. II mandato ricevuto da Valentin era in sostanza quello di riorganizzare e ripulire la struttura e l'immagine dell'amministrazione locale ma, per l'esecuzione del compito, l'incaricato spese una tale quantità di denaro per macchine, edifici, la sua nuova casa con relativa mobilia, mobili per gli uffici (progettati e realizzati appositamente), da rendere più gravoso l'onere rispetto al potenziale risultato; dovette approfittare delle confische dei beni e sui prodotti agricoli per trovare i fondi necessari. Infine l'alacre lavoro di "allestimento" di strutture adeguate e rispettabili distolse molte delle attenzioni necessarie al coordinamento delle attività produttive del distretto.

Frank. Resoconto del HSSPF Krüger sulla situazione nel GG nel 1943, BAB, NS 19/2664, pp. 6-10.

70 Lettera di Karl Valentin al Ministero della Giustizia del Reich del 7 maggio 1938, BAB (ehem. BDC), SA, Valentin, Karl, $1^{\circ}$ agosto 1895.

${ }^{71}$ II 17 settembre 1942, l'amministrazione interna di Ostrów Mazowiecka ordinò l'arresto, eseguito dal capo locale della Gendarmerie Pfüttner, e la deportazione di Moses Seemann al campo di Treblinka, avvenuta il 18 settembre, Doc 141 del 17 settembre 1942, in Friedrich, 2014, p. 440, nota 2.

72 Protocollo della riunione del $1^{\circ}$ aprile 1942, Archiwum Państwowe w Warszawie (APW), KHm Ostrów 75, p. 8.

${ }^{73}$ Protocollo della riunione del 28 aprile 1942, in APW, KHm Ostrów 75, p. 23. 
Inoltre, con parte dei soldi ricavati dalle ulteriori confische, dopo la rivendita dei prodotti agricoli al mercato nero, Valentin fece realizzare delle lussuose scarpe per la moglie. II denaro raccolto non lo teneva nella sede istituzionale, ma in una cassaforte nel suo appartamento, trattenendone una cospicua parte per gli abbellimenti e le ristrutturazioni dell'abitazione e della sede del capo-distretto, mentre pochi erano destinati agli stipendi dei dipendenti. Arrestato e processato, la corte lo condannò a tre anni di detenzione e una multa di 5000 złoty. Ciononostante, la sua attività come riorganizzatore dell'amministrazione locale venne addirittura lodata da alcuni esponenti delle SS, in particolare dalle Waffen-SS. Ernst Kaltenbrunner per esempio, dopo aver chiesto una "speciale riabilitazione» per Valentin nelle Waffen-SS (provvedimento che gli permise di uscire di galera nell'ottobre 1944 e usufruire di un permesso di "lavoro al fronte»), imputò la causa della condotta del funzionario all'influenza esercitata da alcuni elementi ambientali, come «in primo luogo [...] le condizioni caotiche [della] vita economica del Governatorato generale ${ }^{74}$. In esecuzione della sentenza della Corte speciale, Karl Valentin fu espulso dal partito il 18 marzo 1943.

La distruzione e la politica predatoria attuata su larga scala dagli occupanti consentirono l'arricchimento personale di molti gerarchi e funzionari a spese della popolazione, mediante il controllo sui commerci e sul fiorente mercato nero ${ }^{75}$. La commistione tra gerarchi e attività corruttive necessitava di un pretesto di natura politica, o per meglio dire di dottrina razziale: una sorta di "comportamento della razza superiore" tedesca sui locali Untermenschen. Un fattore che consenti maggiori margini di guadagno nelle attività illegali fu il numero, relativamente ridotto, di funzionari di comando presenti sul territorio ${ }^{76}$ : «La ricerca del profitto, la cooperazione economica reciprocamente vantaggiosa e gli interessi comuni erano le principali forze integratrici, insieme al terrore [esercitato] d[a]lla polizia, che tennero insieme il Generalgouvernement durante la guerra ${ }^{77}$.

In molti distretti locali si stabilirono i Landkommissariate e Stadtkommissariate, organi amministrativi non autonomi ma dipendenti dagli uffici centrali delle contee. Nelle sedi locali delle amministrazioni c'era sempre un polacco o un ucraino in regime di "autonomia amministrativa": i tedeschi finsero di considerare le minoranze facendole partecipare alla gestione del territorio, sfruttando invece i locali che lavoravano sottopagati e spesso vessati dai "colleghi" occupanti; vi era

\footnotetext{
${ }^{74}$ Il capo della Sicherheitspolizei e del SD presso il Ministero della Giustizia del Reich, ottobre 1944, in IPN, Regierung des GG I/5238, p. 83; Cancelleria del capo del NSDAP, Ufficio per le domande di grazia presso il governo del GG, Dipartimento di Giustizia, in merito alla domanda di grazia di Karl Wilhelm Valentin, 3 giugno 1944, IPN, Regierung des GG I/5238, p. 63. Non ci sono documenti che testimoniano l'esito della procedura per la richiesta di grazia.

${ }^{75}$ Cfr. Bajohr, 2001, p. 76.

${ }^{76}$ Bajohr, 2001, p. 76; cfr. Pohl, 1997, p. 302.

${ }^{77}$ Gross, 1979, p. 159.
} 
comunque una certa autonomia per gli slavi, specialmente per gli ucraini. I Consigli ebraici (Judenräte) erano al contrario solo gli esecutori degli ordini tedeschi, tanto che la popolazione del ghetto vedeva spesso il Presidente del Consiglio ebraico addirittura come un referente diretto dei nazisti; per questo molti presidenti venivano criticati, disprezzati e minacciati dai propri correligionari (l'esempio più chiaro fu quello di Adam Cerniakow, presidente del Judenrat di Varsavia).

L'amministrazione occupante avrebbe dovuto funzionare secondo il principio dell'unità amministrativa; il principio di competenza e le amministrazioni speciali dovevano essere abolite in tutti e tre i livelli dell'amministrazione statale, ossia governo centrale, distrettuale e di contea o distrettuale locale, e combinate sotto la guida unitaria di una sorta di figura manageriale pubblica, il Governatore (più chiaramente espressa col termine di Verwaltungsführer). Frank intendeva rafforzare il ruolo dei capidistretto, che costituivano secondo lui la spina dorsale dello stato e sui quali andava impostata l'efficienza burocratica e amministrativa, al fine di superare il dualismo stato/partito e velocizzare i processi decisionali, con la nomina di personalità forti dotate di grande assertività e capaci di iniziativa personale. Le inadeguatezze strutturali e personali dei dipendenti e dei dirigenti della pubblica amministrazione del GG determinarono il fallimento del tentativo di imporre l'unità tra le varie anime del settore ${ }^{78}$.

L'apparato burocratico dei distretti e la sede centrale a Cracovia si gonfiavano sempre più mentre i Kreise minacciavano di collassare a causa della penuria di personale. La nuova suddivisione amministrativa limitò i danni al sistema ma non causò un miglioramento duraturo, poiché mancavano le condizioni strutturali per consolidarsi. Il governo di Cracovia ridusse le 72 contee polacche esistenti allo scoppio della guerra fino ai 40 distretti amministrativi locali più sei cittadini del $1^{\circ}$ gennaio 1940 . Il risultato fu una gestione della rete amministrativa strutturata su maglie molto più larghe rispetto al Reich. Rispetto ai Landkreisen tedesche, i Kreise del GG erano di regola svariate volte più grandi: la contea più piccola si estendeva su una superficie di 939 kmq, la più grande misurava 4506 kmq e la media era di 2551 kmq; la popolazione andava dai 95.685 ai 550.405 abitanti, con una media di 253.000 abitanti. Nel Reich, la superficie media di una contea era di 600 kmq e la popolazione media si attestava sui 58.000 abitanti circa ${ }^{79}$.

La suddivisione del personale delle contee e dei distretti cittadini fu modificata parecchie volte nel corso della guerra: come nel caso dell'ordinanza dell'aprile 1941, che determinò l'accorpamento delle contee di Skierniewice e Łowicz.

${ }^{78}$ DTB 19 gennaio 1940, BAB, R 52 II/225, p. 31. Riferimenti anche in Majer, 1981, pp. 487-96.

${ }^{79}$ Cfr. du Prel, 1940, pp. 88, 99-100, 107, 111, 113, 115, 116, 120, 126, 128, 130, 133, 134, $139,170-7,181,207-13$ (i dati sono corrispondenti ai valori demografici e geografici dello stato nei primi 5-7 mesi circa di amministrazione). Con l'annessione della Galizia, i valori sono lievemente cresciuti, mantenendo comunque la distinzione col Reich sul piano delle proporzioni. 
Dopo l'attacco all'Unione Sovietica e l'annessione del Distrikt Galizien una parte del personale del distretto di Cracovia fu trasferita e si applicarono le regole per l'accorpamento di distretti non più necessari e per la creazione di nuovi soggetti amministrativi, come nel caso delle contee di Krosno e Przemyśl. Nel Distrikt Galizien vi furono dapprima quindici contee, poi ridotte a dodici. Anche lo status amministrativo delle città mutava velocemente: oltre alle quattro città capoluogo di distretto (Distrikthauptstädte - Varsavia, Radom, Cracovia, Lublino), anche Tschenstochau (Czestochowa) e Chełm erano guidate all'inizio dalle Stadthauptmannschaften (di fatto le autorità amministrative "autonome" di ogni distretto cittadino).

A metà del luglio 1940, in attuazione dell'accordo di spartizione germano-sovietico sul territorio polacco, la cittadina di Przemyśl assunse lo status di Kreisstadt; il 2 settembre dello stesso anno Kielce, storica città capoluogo del voivodato della Santacroce, divenne la settima Stadthauptmannschaft, numero salito a otto con l'annessione del Distrikt Galizien. Questi movimenti, poveri di motivazioni pratiche ma dettati dalla necessità di mantenere equilibri politici interni alle gerarchie naziste, arrivarono a stabilizzarsi verso la metà del 1943; dopo di che, fino alla fine del GG rimasero 53 Kreishauptmannschaften (gruppi di comando locali) e 6 Stadthauptmannschaften (gruppi di comando cittadini) ${ }^{80}$.

Il potere delle Kreishauptleute era sostanziale e la loro competenza giurisdizionale era ampia; per cercare di limitare i conflitti tra istituzioni diverse e all'interno della stessa istituzione, dall'estate 1940 la figura del capo partito sul territorio coincise con quella del Standortführer, rendendo quindi il funzionario locale di riferimento meno indipendente e più controllabile direttamente da Berlino. ॥ Kreishauptmann aveva la responsabilità dell'attuazione delle direttive e disposizioni adottate dal governo centrale di Cracovia; egli doveva essere "I'unico rappresentante del Governatorato generale» nel distretto locale ed era subordinato al Governatore ${ }^{81}$. De facto però, date le circostanze spesso precarie in cui operava l'amministrazione centrale, «il governatore distrettuale locale [si trovò spesso ad] eseguire [...] in modo spesso indipendente provvedimenti e disposizioni, in modo che corrispond[essero] agli orientamenti politici del Governatore generale e garantis[sero] il raggiungimento degli obiettivi riconoscibili dalla sua azione. Grandi dunque [furono] le aspettative nei confronti del governatore distrettuale locale, così come di ogni suo collaboratore ${ }^{82}$.

In generale, le sfere di competenza di Kreis- e Stadthauptleute dovevano essere regolate dall'alto, con una moltitudine di normative, vincoli e limitazioni (Verordnungsflut) che lasciavano all'Hauptmann la responsabilità di definire le priorità

\footnotetext{
${ }^{80}$ du Prel, 1940, pp. 88, 101, 147-8, 207; Relazione sulla struttura del GG fino al $1^{\circ}$ luglio 1940, BAB, R 52 II/247, p. 40.

${ }^{81} \mathrm{Cfr}$. Secondo ordine sulla struttura dell'amministrazione del GG (Ordinanza sull'unità dell'amministrazione) del $1^{\circ}$ dicembre 1940, in Nolzen, 1997, pp. 247-75 (qui pp. 254-5). ${ }^{82}$ Gollert, 1942, p. 88.
} 
per l'esecuzione ${ }^{83}$. Queste problematiche si presentarono anche nel nuovo Distrikt Galizien, dove i Kreishauptleute conquistarono un ancor più ampio margine di manovra: "potevamo fare ciò che volevamo ${ }^{84}$, dichiarò tempo dopo Wilhelm Rebay von Ehrenwiesen in una intervista ${ }^{85}$.

Frank non mancò mai di elogiare le capacità di adattamento dei dirigenti locali, anche nei frequenti casi di abuso di potere e uso eccessivo di metodi repressivi, poiché valutava le azioni rispetto al raggiungimento dell'obiettivo prestabilito, qualificando la violazione come «typisch östlich illegale Art» (tipica fattispecie illegale orientale) ${ }^{86}$.

\section{Quando il "diritto" è allo sbando}

Le responsabilità dei capi locali, nell'esercizio delle loro funzioni, non venne regolata da una legge apposita, ma si applicarono in deroga le norme sulla disciplina dei singoli settori dell'attività amministrativa. I settori produttivi erano spesso per intero nella loro sfera di competenza: il rafforzamento del potere tedesco sul territorio, la riscossione di quote di raccolto in agricoltura, il mercato del lavoro e il controllo diretto sulla manodopera locale, lo sfruttamento delle risorse minerarie ed energetiche, il controllo sul sistema economico e produttivo nonché la sorveglianza sulla Selbstverwaltung di polacchi, ebrei e ucraini. Inoltre i Kreishauptleute dovevano occuparsi di passaporti, notifiche, controllo dei prezzi, lotta al contrabbando, stato sociale per tedeschi e polacchi, trasporti, organizzazione e supervisione delle strutture sanitarie e del personale medico. Rispetto al settore agricolo, essi avevano la funzione di monitoraggio della fornitura e della distribuzione di sementi e fertilizzanti, gestione del magazzino delle quote agricole. In più la Kreishauptmannschaft gestiva i controlli nelle scuole elementari e negli istituti tecnici e forniva l'assistenza culturale per tutti i Volksdeutsche ${ }^{87}$.

${ }^{83}$ DTB 19 gennaio 1940, in BAB, R 52 II/225, pp. 29-30; Präg, Jacobmeyer, 1975, p. 415 (nota del 16 ottobre 1941).

${ }^{84}$ Präg, Jacobmeyer, 1975, p. 415 (nota del 16 ottobre 1941). A questo proposito è indicativa una vicenda che vide protagonista Friedrich Gollert. II Dr. Gollert, capo del Dipartimento di Giustizia dell'Ufficio del governatore di Varsavia, fece arrestare, tra il 10 e il 12 luglio 1940, 80 avvocati polacchi con l'accusa di aver sostenuto che anche gli avvocati ebrei dovevano essere ammessi all'esame di abilitazione alla professione, e li fece rinchiudere nella prigione di Pawiak. I prigionieri furono spediti ad Auschwitz nel settembre 1940. Cfr. Iranek-Osmecki, 2009, pp. 119-20, https://ipn.gov.pl/pl/publikacje/ ksiazki/12641,Kto-ratuje-jedno-zycie-Polacy-i-Zydzi-19391945.html (11-06-2021).

${ }^{85}$ Giurista e burocrate tedesco, Ehrenwiesen fu nominato Kreishauptmann del distretto locale di Kamionka Strumiłowa, nel Distrikt Galizien; in Pohl, 1997, p. 419.

${ }^{86}$ DTB 16 ottobre 1941, in BAB, R 52 II/239, p. 52.

${ }^{87}$ Cfr. Primo decreto sull'istituzione dell'amministrazione dei territori polacchi occupati del 26 ottobre 1939, in Documenta Occupationis (Doc. Occ.) VI, p. 56, http://www. iz.poznan.pl/archiwum/documenta-occupationis/, (24-06-2021); Kreishauptmann Heinz Gustav Albrecht, esempi di amministrazione di una Kreishauptmannschaft, IPN, NTN 381, 
Inizialmente non c'era uno schema organizzativo unificato; ogni Kreishauptmann poteva costruire la propria autorità a sua discrezione e in base alle condizioni ambientali incontrate. Alla fine del marzo $1941 \mathrm{fu}$ introdotta la regolamentazione che consentiva l'aggregazione o l'integrazione di autorità speciali nell'amministrazione fino al $1^{\circ}$ maggio. Furono aggregati l'ufficiale sanitario, l'ispettorato della motorizzazione, l'ufficio di stato civile per cittadini del Reich, l'organismo di controllo sui prezzi, gli ispettori distrettuali locali nel settori agricolo e dell'istruzione, l'ispettorato finanziario (Finanzinspekteur), l'ispettore territoriale (Landinspektion) e quello addetto alla gestione delle risorse idriche (Wasserwirtschaftsinspektion), l'ufficio del lavoro (Arbeitsamt), l'ufficio di controllo sulla silvicoltura (Forstaufsichtsamt) e l'organismo di controllo sui cantieri in costruzione (Strombauinspektion). In più vennero subordinate al Kreishauptmann autorità guidate da "non tedeschi": I'ufficio topografico della contea (Kreisvermessungsamt), l'ambulatorio veterinario (Amtstierarzt), l'ufficio di verifica di pesi e misure (Eichamt), l'autorità dei terreni e delle acque. Nel caso di integrazione tra gli uffici era lo stesso Kreishauptmann il sovrintendente capo della sua contea; qualora si fosse proceduto ad un'azione di accorpamento, la responsabilità era in mano ad un ufficio indipendente al quale il Kreishauptmann aveva facoltà di dare istruzioni vincolanti sull'operato (Weisungsrecht) nella misura necessaria alla salvaguardia di una linea politica uniforme, o per bilanciare i punti di vista contrastanti di diversi rami dell'amministrazione. Nell'aprile 1941 un'ordinanza amministrativa disciplinò infine uniformemente il profilo dei servizi dei Kreishauptleute; di conseguenza l'autorità risultò divisa in quattro settori: amministrazione interna (Innere Verwaltung), economia (Wirtschaft), alimentazione e agricoltura (Ernährung und Landwirtschaft), istruzione (Schulamt) ${ }^{88}$.

Nel luglio 1943 la normativa subì un ulteriore cambiamento. La polizia locale divenne l'ufficio per gli affari di polizia all'interno di Kreishauptmannschaften e Stadthauptmannschaften; così il capo della polizia locale si trovava sotto il controllo del Kreishauptmann, come organo esecutivo di polizia.

Per lo svolgimento dell'opera di amministrazione le autorità territoriali locali e cittadine si servirono di piccole Gendarmeriezüge (compagnie locali di pubblica sicurezza). In ogni contea una compagnia era di stanza in partenza con 20-25 uomini, per poi arrivare anche a 30-40 elementi. La Gendarmerie svolgeva inizialmente una semplice attività di polizia, senza alcuna competenza sulle misure disciplinari. II 17 ottobre 1941 divenne operativa la cosiddetta "autorità incondizionata" dei capi cittadini e dei capi distrettuali locali sulla Gendarmerie ${ }^{89}$, che venne inoltre subordinata alle autorità polacche di polizia attive ancora sul ter-

pp. 111-33.

${ }^{88}$ Circolare amministrativa $\mathrm{n} .4$ del regolamento relativo all'unità dell'amministrazione, con efficacia a partire dal $1^{\circ}$ dicembre 1940, 18 aprile 1941, AAN, Regierung des GG, 9c/3, pp. 54-6.

89. Cfr. Präg, Jacobmeyer, 1975, pp. $425-7$ (nota del 17 ottobre 1941). 
ritorio (anche se la subordinazione era solo di facciata e determinata per ragioni corporative e politiche interne agli organismi di governo occupanti), con compiti di lotta alla criminalità comune e di pattugliamento principalmente dei quartieri cittadini. La polizia polacca venne sfruttata al pari dei lavoratori coatti polacchi dell'industria e alla manodopera contadina. In molti casi i tedeschi utilizzavano i poliziotti autoctoni per alleggerire il carico di lavoro delle SS e della polizia locale per la deportazione e l'uccisione degli ebrei dei ghetti.

Le contee e le amministrazioni cittadine avevano anche il controllo del Sonderdienst, il servizio speciale di protezione dei Volksdeutschen che, creato nell'autunno 1939 reclutando i membri dalla minoranza tedesca, partecipò nei primi mesi alle uccisioni sommarie compiute dagli occupanti. II Sonderdienst venne chiamato da Frank il 6 maggio 1940 in aiuto delle Kreishauptleute e renderle indipendenti dalla Gendarmerie nell'esercizio delle loro funzioni. Grazie al Sonderdienst, la Zivilverwaltung avrebbe dovuto riprendersi il controllo di aree lasciate incustodite per carenza di personale e dissidi con la polizia (che non sempre era disposta ad assecondare gli amministratori locali andando a sopperire alle loro lacune normative e di organico). Ogni squadra, composta da 20-30 unità di Volksdeutschen in ogni Kreis, rispondeva al Kreishauptmann che ne faceva principalmente uso per: la lotta al contrabbando (Bekämpfung des Schleichhandels), la riscossione delle quote di raccolto dei prodotti agricoli (Erfassung der Kontingente), imposte (Eintreibung von Steuern), tasse e contravvenzioni (Gebühren und Strafgeldern), nonché il servizio di guardia della sede della Kreishauptmannschaft, del Kreishauptmann e dell'eventuale campo di detenzione locale ${ }^{90}$.

II numero di poliziotti ucraini variava da contea a contea e oscillava dai 100 ai 400 circa. Nelle contee con un distaccamento della Sicherheitspolizei (Sipo) c'erano almeno 20 agenti operativi, sui quali però il Kreishauptmann non aveva alcun controllo. Vi erano poi formazioni come il Werkschutz (sicurezza aziendale), Forstschutz (sicurezza forestale - settore della silvicoltura), Bahnschutz (sicurezza ferroviaria), con ruoli minori e circoscritti. Nel complesso si può stimare una presenza di 200-500 persone appartenenti ai servizi di sicurezza, suddivise nelle varie categorie e formazioni di appartenenza, escludendo la Wehrmacht, i Polizeibataillone e le $\mathrm{SS}^{91}$.

Fino alla fine dell'estate del 1940 le contee e le amministrazioni cittadine (ad esclusione dei centri principali) non erano formalmente in possesso di alcun potere punitivo di natura giuridica; venivano attuate solo misure arbitrarie stabilite dagli Hauptmänner, come nei già citati casi di minacce, percosse, torture, ese-

\footnotetext{
${ }^{90}$ DTB 24-25 febbraio 1940, BAB, R 52 II/230, p. 14; Präg, Jacobmeyer, 1975, p. 352 (nota del 8 aprile 1941); Broszat, 1961, p. 77.

${ }^{91}$ Cfr. Chodakiewicz, 2004, pp. 105-32. Una menzione a parte va fatta per i Polizeibataillone: composti da vari membri delle SS dalla non sempre spiccata appartenenza ideologica, furono gli esecutori di non specificate "misure di polizia» (sicherheitspolizeiliche Maßnhmen) contro gli ebrei nei territori occupati. Vedi Browning, 2003, pp. 118-24.
} 
cuzioni capitali, danneggiamenti a proprietà private. Solo il 13 settembre 1940 venne emanato il regolamento sui procedimenti amministrativi che regolava le unità amministrative locali; i Kreishauptmänner poterono così imporre sanzioni pecuniarie fino ai 1000 zloty o in alternativa comminare pene fino ai tre mesi di reclusione $^{92}$.

All'inizio della primavera del 1940 alcuni capi contea del distretto di Lublino allestirono campi di detenzione e di lavoro per i polacchi e gli ebrei che avessero trasgredito le loro disposizioni o che non si fossero conformati adeguatamente ad esse. Tra i soggetti colpiti dal provvedimento, gli agricoltori che ottemperavano poco o per nulla alle richieste sulle quote di consegna; l'autorità civile spesso inseriva tra gli "ospiti" dei campi anche contrabbandieri e scansafatiche (Arbeitsscheue). I detenuti dovevano svolgere lavori pesanti, ritenuti un metodo adeguato di trattamento «per contribuire straordinariamente al naturale equilibrio dei polacchi, e dare la necessaria enfasi per obbedire agli ordini» ${ }^{93}$. Ogni azione venne svolta senza alcuna copertura normativa, elemento trascurato dal governo di Cracovia; anche i governatori di Varsavia e di Radom tollerarono e introdussero molte procedure non previste dalla normativa, come la creazione dei campi di lavoro non formalmente autorizzati. II Governatore tentò in più occasioni di dare una base giuridica all'azione amministrativa dello Stato in tutti i suoi settori, per giustificarne e "nobilitarne" gli atti al fine di creare una sorta di "normalizzazione" dell'applicazione del sistema amministrativo nazista sul territorio ${ }^{94}$. Le finalità erano ovviamente di tipo pratico, con due esempi su tutti che ne spiegano la portata: il primo riguardò l'intenzione di Frank di apparire un Governatore (apparentemente) morbido e dai modi "equilibrati" per gestire la popolazione polacca sul territorio, tramite l'utilizzo di una decretazione (rigorosamente di tipo personale) che disciplinasse un trattamento minimamente rispettoso, al fine di poter «dispo[rre] di [un adeguato] person[ale] per [la] costitu[zione] [e il funzionamento di] un apparato [amministrativo]»; il secondo invece mirò al mantenimento di una "buona reputazione" dei "governanti" tedeschi, così da far "desiderare [al]le altre nazioni di [finire] sotto la protezione tedesca»" ${ }^{95}$.

Nel distretto di Varsavia il Kreishauptmann Gramß istituì nel giugno 1941 un campo di lavoro a Treblinka nella contea di Sokołów (rinominato in seguito Treblinka I, da non confondere col successivo campo di sterminio, allestito l'anno successivo in un luogo differente). Gramß volle prevenire gli intoppi burocratici

\footnotetext{
92 Ordinanza sulla procedura penale amministrativa nel Generalgouvernement del 12-13 settembre 1940, Verordnungsblatt des Generalgouvernements (VBGG) 1940, pp. 300-2, http://dlibra.umcs.lublin.pl/dlibra/publication?id=11980\&tab=3 (11-06-2021), archivio online della UMCS Digital Library di Lublino. Vedi anche Musial, 1999, pp. 49-51; Majer D., 1981, passim.

${ }^{93}$ Gollert, 1942, p. 95.

${ }_{94}$ DTB 13 aprile 1942, BAB, R 52 II/243, pp. 32-3; Musial, 1999, pp. 53-4.

${ }^{95}$ Präg, Jacobmeyer, 1975, pp. 146-9.
} 
per i lavori di costruzione e assegnò il comando del campo a Theo van Eupen, un funzionario del suo ufficio. Poco più tardi fu lo stesso governatore distrettuale Fischer a stabilire l'apertura dei campi di detenzione penale nel distretto di Varsavia; oltre al governatore, SSPF, Kreis- e Stadthauptleute fecero internare oppositori nei campi istituiti "illegalmente"; la costruzione del campo di lavoro era finalizzata anche a scoraggiare la stampa clandestina e le campagne di affissione dei dissidenti polacchi. Il campo di Treblinka poteva contenere fino a 3000 prigionieri ma, dal momento che molti degli internati avrebbero potuto dare un contributo al settore tessile, specialmente per la produzione di divise per l'esercito, i Kreis-e Stadthauptleute furono costretti a limitarsi negli arresti, al fine di «poter dare ancora a Varsavia un numero sufficiente di asociali e fannulloni polacchi» ${ }^{96}$. Oltre al lavoro nelle officine, i prigionieri venivano utilizzati per la costruzione di strade, nelle cave di ghiaia e nella silvicoltura; fino alla chiusura del campo nell'agosto 1944 vi lavorarono 10.000 prigionieri ${ }^{97}$.

L'esempio del Kreisarbeitslager venne seguito da molti amministratori locali durante il 1941; solo il distretto di Cracovia costituì un'eccezione, nella quale i detenuti polacchi finivano nelle carceri ordinarie o in altre strutture di detenzione. Nel Distrikt Galizien gli agricoltori che non versavano le quote venivano internati in strutture delle SS, diverse dai campi di lavoro ${ }^{98}$.

Molti gerarchi, impauriti dalle prospettive nel dopoguerra, Governatore compreso (il quale anzi fu precursore in questo cambio di rotta), iniziarono un patetico e spesso insincero processo di riavvicinamento verso la popolazione polacca per cercare di ripulirsi la coscienza e (soprattutto) salvarsi la vita e per preservare una posizione all'interno dell'amministrazione anche dopo la guerra ${ }^{99}$. Da questi spesso impalpabili tentativi rimase piuttosto distante la condotta nelle attività economiche, che risultavano in ogni caso l'elemento più importante a livello amministrativo della Zivilverwaltung.

\section{Conclusioni}

A proposito dell'applicazione della Verwaltungskunst ("arte" dell'amministrazione, intesa come insieme di grandi abilità di tipo "manageriale"), le ambizioni del Governatore uscirono ridimensionate da un quadro normativo incompleto e da modalità di azione basate più che altro sul potere personale dei singoli capi distretto locali.

\footnotetext{
${ }^{96}$ Riferimenti su casi di internamento, come quello di Moses Seemann dalla contea di Ostrów, in Friedrich, 2014, p. 440.

${ }_{97}$ Wojtczak, 1975, pp. 117-135, in http://iucat.iu.edu/catalog/2145349 (11-06-2021), archivio online dell'Indiana University.

${ }^{98}$ DTB 13 luglio 1942, BAB, R 52 II/242, p. 65.

${ }^{99}$ Kotula, 1999, p. 89. Riguardo al Governatore ricordo il suo Im Angesicht des Galgens..., passim, che riassume perfettamente la maturazione di un pensiero di (probabile) pentimento e paura per le azioni commesse.
} 
Tutti i Kreise e le amministrazioni cittadine del GG costituirono più di semplici cinghie di trasmissione nell'architettura dello stato. Esse infatti rappresentarono, ciascuna in misura diversa, le varie forme di personalizzazione del potere e abuso di metodi coercitivi all'interno della già "peculiare" macchina amministrativa guidata da Frank.

La portata distruttiva con cui fu condotta l'attività di governo delle singole aree non fu solamente il risultato di carenze strutturali, ma venne costruita e "vissuta" volontariamente dai funzionari locali. Frank cancellò la visione centralista dello stato alla quale parve ispirarsi nei primi mesi di vita del GG, mostrando non poca incoerenza rispetto agli stessi principi guida della dottrina nazionalsocialista, da lui elaborati o comunque sottoscritti, sull'importanza di un indirizzo unico e delineato, sul quale concentrare tutte le energie dello stato. Rimase la "sacralità" del Füherprinzip, ma la dispersione del potere tra i molti livelli amministrativi, di cui i vari signori locali approfittarono per fornire ulteriori interpretazioni sull'applicazione della «volontà del Führer» e per arricchirsi e fare carriera attraverso la malversazione e la carenza di scrupoli, determinò l'esito fallimentare dell'intera esperienza "governatorale".

Con l'esperienza del GG, risultò chiaro anche ai giuristi più rigorosi che la codificazione e la effettiva istituzionalizzazione del fenomeno nazionalsocialista erano obiettivi impossibili da raggiungere. II richiamo alla "forza del diritto» di Frank può essere inteso, oltre che un modo con cui il despota tentò di deresponsabilizzarsi di fronte al mondo rispetto alle atrocità commesse, anche come il formale riconoscimento dell'utopia del «diritto comunitario tedesco» da parte del giurista, del conseguente fallimento dell'esperienza di governo del Governatore, e della incapacità dell'uomo di raggiungere quel livello di autorevolezza e di credibilità per arginare e superare le proprie insicurezze personali. II Governatorato generale amplificò dunque, nella sua peculiarità, tutti i difetti e le storture del sistema di stato e di teoria giuridica del nazionalsocialismo.

La costruzione della struttura amministrativa fu decretata dall'alto, ma ebbe effettivamente luogo dal basso. In molti distretti locali, specialmente nelle vaste zone rurali del paese, la popolazione slava si trovò a trattare quasi esclusivamente con i livelli più bassi dell'amministrazione occupante, poiché proprio ai capi distretto locali, ai capi delle rispettive Gendarmerie, ai distaccamenti della polizia e del SD fu sostanzialmente demandato l'onere di tradurre le direttive generali e i regolamenti in azioni e comportamenti funzionali al raggiungimento degli obiettivi dello stato. Hitler infatti non era interessato ai risvolti giuridici o istituzionali dei quali parlava Frank, ma vide il GG come la «pattumiera sociale del Reich», da sfruttare per ragioni razziali ed economiche. 
Fonti

AAN (Archiwum Państwowe w Warszawie), KHm Ostrów 75

AAN, Regierung des GG, $9 c / 3$

BA (Bundesarchiv) R $43 \mathrm{II} / 1648$

BA R 43 II/958

BAB (Bundesarchiv Berlin), R 43, II/141

BAB, NS 19/2664

BAB (ehem. BDC), SA, Valentin, Karl, $1^{\circ}$ agosto 1895

$B A B, R 52 \mathrm{II} / 225$

$B A B, R 52 I / 247$

$B A B, R 52 I / 239$

$B A B, R 52 \mathrm{II} / 230$

$B A B, R 52 \mathrm{II} / 243$

$B A B, R 52 \mathrm{II} / 242$

BAL (Bundesarchiv Ludwigsburg), B 162/Dok-Slg. Polen, Ordn. 344

BAL, B 162/202 AR-Z 76/61, parte 2

Doc. Occ. (Documenta Occupationis) VI

DTB (Diensttagebuch), IfZM, Fb 105/30

DTB, IfZ, Fb 105/1, Bl. 40

IPN (Instytut Pamięci Narodowej) BU-2586-329

IPN, NTN 381

IPN, Regierung des GG I/5238

RGBI. (Reichsgesetzblatt), 1934, I

RGBI., 1943, I

RGBI., 1933, I

\section{Bibliografia}

Baedeker K., 1945: Das Generalgouvernement: Reisehandbuch, Leipzig, Baedeker

Bajohr F., 2001: Parvenus und Profiteure: Korruption in der NS-Zeit, Frankfurt am Main, S. Fischer

Best W., 1938: Neuordnung des Polizeirechts, in "Jahrbuch der Akademie für Deutsches Recht", vol. 5, pp. 44-50

Best W., 1941: Die deutsche Polizei, Darmstadt, Wittich

Böckenförde E. W. (ed.), 1985: Staatsrecht und Staatsrechtslehre im Dritten Reich, Heidelberg, Müller

Broszat M., 1961: Nationalsozialistische Polenpolitik 1939-1945, Stuttgart, Deutsche Verlags-Anstalt

Browning C., 2003: Nazi policy, Jewish workers, German killers, Cambridge, Cambridge University Press 
Chodakiewicz M. J., 2004: Between Nazis and Soviets. Occupation politics in Poland 1939-1947, Lanham, Lexington Nooks

Du Prel M. F., 1940: Das deutsche Generalgouvernement Polen: ein Überblick über Gebiet, Gestaltung und Geschichte, Krakau, BVO

Echterhölter R., 1970: Das öffentliche Recht im nationalsozialistischen Staat, fonti e interpretazioni della storia contemporanea 16/II, Stuttgart, Deutsche VerlagsAnstalt

Engelking B., Leociak J., 2009: The Warsaw Ghetto: a Guide to the Perished City, New Haven, Yale University Press

Frank H., 1953: Im Angesicht des Galgens. Deutung Hitlers und seiner Zeit auf Grund eigener Erlebnisse und Ernkenntnisse, München, F.A. Beck

Friedrich K. P., Heim S., 2013: Die Verfolgung und Ermordung der europäischen Juden durch das nationalsozialistische Deutschland 1933-1945, Band 9, Polen: Generalgouvernement August 1941-1945, München, De Gruyter

Friedrich K. P., 2014: Polen: Generalgouvernement, August 1941-1945, Munich, Oldenburg Verlag

Gollert F., 1942: Warschau unter deutscher Herrschaft: deutsche Aufbauarbeit im Distrikt Warschau. Im Auftrage des Gouverneurs des Distrikts Warschau, SA-Gruppenführer Dr. Ludwig Fischer, unter Benutzung amtlicher Unterlagen, Burgverlag Krakau

Gross J.-G., 1979: Polish society under German occupation: the Generalgouvernement, 1939-1944, Princeton (N.J.), Princeton University Press

Harten H.-C., 1996: De-Kulturation und Germanisierung: Die nationalsozialistische Rassen- und Erziehungspolitik in Polen 1939-1945, Frankfurt a. M., CampusVerlag

Herbert U., 1996: Best. Biographische Studien über Radikalismus, Weltanschauung und Vernunft 1903-1989, Bonn, Dietz

Huber E. R., 1935: Das Staatsoberhaupt des Deutschen Reiches, in „Zeitschrift für die gesamte Staatswissenschaft", vol. 95, pp. 202-29

Huber E. R., 1941: Reichsgewalt und Reichsfürung im Kriege, in „Zeitschrift für die gesamte Staatswissenschaft", vol. 101, pp. 530-79

Hubert P., 1992: Uniformierter Reichstag. Die Geschichte der PseudoVolksvertretung 1933-1945, Beiträge zur Geschichte des Parlamentarismus und der Politischen Parteien 97, Düsseldorf, Droste

Iranek-Osmecki K., 2009: Kto ratuje jedno życie... Polacy i Żydzi 1939-1945 (Chi salva una vita... Polacchi ed ebrei 1939-1945), Warszawa, IPN

Koellreutter O., 1938: Deutsches Verfassungsrecht. Ein Grundiss, Junker und Dünnhaupt

Korte H., 1942: Führererlass und Führerverordnung als Mittel der Führergewalt, 
in „Deutsche Verwaltung", vol. 19, pp. 473-6, 498-501

Kotula F., 1999: Losy Żydów rzeszowskich 1939-1944: kronika tamtych dni, Rzeszów, Mitel

Madajczyk C., 1987: Die Okkupationspolitik Nazideutschlands in Polen 19391945, Berlin, Akademie-Verlag

Majer D., 1981: "Fremdvölkische" im Dritten Reich: ein Beitrag zur nationalsozialistischen Rechtssetzung und Rechtspraxis in Verwaltung und Justiz unter besonderer Berücksichtigung der eingegliederten Ostgebiete und des Generalgouvernements, Boppard am Rhein, Boldt

Majer D., 1987: Grundlagen des nationalsozialistischen Rechtssystems: Führerprinzip, Sonderrecht, Einheitspartei, Stuttgart, Kohlhammer

Manoschek W., 1995: „Es gibt nur eines für das Judentum-Vernichtung“: das Judenbild in deutschen Soldatenbriefen 1939-1944, Hamburg, Hamburger Edition

Młynarczyk J. A., 2004: Die zerrissene Nation. Die polnische Gesellschaft unter deutscher und sowjetischer Herrschaft 1939-1941, in Mallmann K.-M., Musial B. (ed.), Genesis des Genozids. Polen 1939-1941, Darmstadt, Wissenschaftliche Buchgesellschaft, pp. 145-6

Moll M. (ed.), 2011: Führererlasse 1939-1945, Hamburg, Nikol

Musial B., 1999: Deutsche Zivilverwaltung und Judenverfolgung im Generalgouvernement: eine Fallstudie zum Distrikt Lublin 1939-1944, Wiesbaden, Harrassowitz

Nehring J., 1940: Dienst im Osten, in „Krakauer Zeitung “, 11 giugno, n. 136

Nolzen A., 1997: Der Arbeitsbereich der NSDAP im Generalgouvernement, in Bohn R. (ed.), Arbeitsbereich: Die deutsche Herrschaft in den "germanischen" Ländern 1940-1945, Stuttgart, Steiner, pp. 247-75

Pohl D., 1997: Nationalsozialistische Judenverfolgung in Ostgalizien 19411944: Organisation und Durchführung eines staatlichen Massenverbrechens, München, Oldenbourg

Präg W., Jacobmeyer W. (ed.), 1975: Das Diensttagebuch des deutschen Generalgouverneurs in Polen 1939-1945, Stuttgart, Deutsche Verlags-Anstalt

Ritter G. (ed.), 1952: Conversazioni di Hilter a tavola 1941-1942. Raccolte al suo quartier generale da Henry Picker, Milano, Longanesi

Roth M., 2009: Herrenmenschen. Die deutschen Kreishauptleute im besetzen Polen: Karrierewege, Herrschaftspraxis und Nachgeschichte, Göttingen, Wallstein Verlag

Rüthers B., 1988: Entartetes Recht. Rechtslehren und Kronjuristen im Dritten Reich, München, Beck

Schaefer A., 1985: Führergewalt statt Gewaltenteilung, in Ernst-Wolfgang 
Böckenförde (ed.), Staatsrecht und Staatsrechtslehre im Dritten Reich, Heidelberg, Müller, pp. 89-105

Schwaneberg S., 2009: Eksploatacja gospodarcza Generalnego Gubernatorstwa przez Rzeszę Niemieckq w latach 1939-1945 (Lo sfruttamento economico del Governatorato generale da parte del Reich tedesco nel 1939-1945), in Instytut Pamięci Narodowej (ed.), Pamięć i Sprawiedliwość (memoria e giustizia), vol. 1, n. 14, Warszawa, pp. 133-54

Weber W., 1942: Führererlass und Führerverordnung, in „Zeitschrift für die gesamte Staatswissenschaft", vol. 102, pp. 101-37

Wojtczak S., 1975: Karny obóz pracy Treblinka I i ośrodek zagłady Treblinka II (II campo di lavoro penale Treblinka I e il centro di sterminio Treblinka II), in „Biuletyn Głównej Komisji Badania Zbrodni Hitlerowskich w Polsce“, vol. 26, pp. 117-135

Zitelmann R., 1992: Hitler-Bild im Wandel, in Karl Dietrich Bracher, Manfred Funke, Hans-Adolf Jacobsen (ed.), Deutschland 1933-1945. Neue Studien zur nationalsozialistische Herrschaft, Bonn, Schriften zur Politik und Zeitgeschichte, pp. 491-506

\section{Sitografia}

https://ipn.gov.pl/pl/szukaj?search=4061521\&sort=2\&order=1\&ile=20 (20-052021)

Jehke R. (ed.), Territoriale Veränderungen in Deutschland und deutsch verwalteten Gebieten 1874-1945, in http://www.territorial.de/gg/opatow/kreish.htm (2005-2021), caricato il 20 agosto 2009

http://jewishsokolow.blogspot.it/2011/08/ernst-gramss.html (16-06-2021)

http://bc.radom.pl/dlibra/plain-content?id=23554 (26-05-2021)

https://ipn.gov.pl/pl/publikacje/ksiazki/12641,Kto-ratuje-jedno-zycie-Polacy-iZydzi-19391945.html (11-06-2021)

http://www.iz.poznan.pl/archiwum/documenta-occupationis/, (24-06-2021)

http://dlibra.umcs.lublin.pl/dlibra/publication?id=11980\&tab=3 (11-06-2021)

http://iucat.iu.edu/catalog/2145349 (11-06-2021) 
